\title{
Geographic Origin and Diversity of Wine Strains of Saccharomyces
}

\author{
Linda F. Bisson ${ }^{1 *}$
}

\begin{abstract}
The availability of genome sequence information from a large collection of strains of Saccharomyces isolated from a variety of geographic regions and ecological niches has enabled a detailed analysis of genome composition and phenotype evolution, the two components of strain diversity. These analyses have also provided a relatively complete depiction of the origins of wine strains. In population genomic analysis, wine strains of $S$. cerevisiae cluster as a highly related group, but one that shows a greater level of phenotypic differentiation than would be predicted based on the level of genomic similarity. Natural and human selection and genetic drift have played roles in the evolution of wine strain diversity. Phenotypic diversity is so extensive that no one strain accurately represents all wine strains with respect to biological properties and fermentation performance. In addition, both commercial and native isolates have been found to carry introgressions, regions of DNA derived from nonhomologous organisms, suggestive of cell fusion events with yeast of different genera and species. Comparative sequence analysis has thus refined our knowledge of yeast lineages and offers explanation for the evolution of phenotypic diversity observed in winery and vineyard populations.
\end{abstract}

Key words: yeast diversity, Saccharomyces, genomic analysis

The yeast Saccharomyces cerevisiae was identified as the agent of beverage fermentation by Louis Pasteur in 1860 (Pasteur 1860). After roughly 150 years of research, our understanding of the biology of this important organism is extensive. The completion of the sequence of the genome of a laboratory strain, S288C, facilitated intensive study of the numbers of genes present in this organism, their functions, and mechanisms of regulation (Oliver et al. 1998). The subsequent sequencing of the genomes of other strains of Saccharomyces from diverse ecological niches and of related and unrelated yeast species has generated novel insights into the origin of the modern wine strain lineage, the evolution of phenotypes and genetic diversity, and the biological plasticity of this important industrial organism (Bullard et al. 2010, Carreto et al. 2008, Cavalieri 2009, Cubillos et al. 2011, Diezmann and Dietrich 2009, Ezeronye and Legras 2009, Fay and Benavides 2005a, 2005b, Legras et al. 2007, Lelandais et al. 2011, Liti et al. 2009, Muller and McCusker 2011, Roberts and Oliver 2010, Schacherer et al. 2009, Spor et al. 2009, Warringer et al. 2011).

Even though different types of tools and analyses have been applied to the investigation of genetic diversity within S. cerevisiae, the single truth that has emerged is that no one strain phenotypically portrays the entire species (Kvitek et al. 2008, Liti and Schacherer 2011). Concerns with use of the

\footnotetext{
'Professor, Department of Viticulture and Enology, 595 Hilgard Lane, University of California, Davis, CA 95616.

*Corresponding author (email: 1fbisson@ucdavis.edu; tel: 530 752-3835; fax: 530 752-0382)

Manuscript submitted Aug 2011, revised Feb 2012, accepted Feb 2012

Copyright (C) 2012 by the American Society for Enology and Viticulture. All rights reserved.

doi: 10.5344/ajev.2012.11083
}

strain S288C as characteristic of the species have arisen because this strain derives from a mosaic lineage constructed in the laboratory (Mortimer and Johnston 1986) that has never been exposed to native environments or to natural selection (Liti and Schacherer 2011, Warringer et al. 2011). This strain shows genetic variation and growth and expression profiles not observed in wild isolates and rarely clusters with other lineages (Warringer et al. 2011). A second important overall conclusion of these studies is that the diversity that exists is well explained by the genetic phenomena that have been characterized in laboratory strains suggesting that genetic cycles, DNA exchange, cell fusion, and spontaneous mutagenesis that have been so successful in the exploitation of Saccharomyces as a model system for research also occur in the wild (Dequin and Casaregola 2011, Lelandais et al. 2011, Sipiczki 2011).

Comprehensive analysis of isolates from diverse geographical areas and from different ecological environments within those areas identified five distinct lineages of Saccharomyces strains: West African, Malaysian, North American, Sake, and European (Liti et al. 2009, Liti and Schacherer 2011). Strains also group by technological origin (Legras et al. 2007). Analysis of isolates of Saccharomyces from throughout the world found that $95 \%$ of the wine, winery, and vineyard isolates were grouped in the same cluster with cider strains (Legras et al. 2007). Bread yeasts in contrast were divided into two different groups (Legras et al. 2007). Among the wine yeast, distinct subgroupings could be identified. The majority of strains belonged to the Central European group but other clusters, Champagne yeast and the UCD522 group, were also evident (Legras et al. 2007). The UCD522 and Champagne clusters cross geographic origin and are due to the influence of human selection in wine production, as progenitors of these strains were likely introduced into these diverse winemaking regions. These analyses have led to the conclusion that 
lineage or parentage and technological use have as much of an influence on strain diversity as geographical region. In contrast, strains of Saccharomyces from nontechnological origins (fruit, soil, oak, and insects) and other species such as $S$. paradoxous that have not undergone rounds of domestication show a stronger clustering by region of isolation (Liti et al. 2009, Liti and Schacherer 2011).

The striking genetic similarity of winery and vineyard isolates has been taken as evidence of a single domestication event followed by dispersal of the domesticated strains (Legras et al. 2007, Sicard and Legras 2011). The first historical evidence of wine production comes from the discovery of tartaric acid in ancient jars of Mesopotamia and suggests a sophisticated wine industry existed in 5400 to 5000 BC (McGovern et al. 1996). The point of origin of the first wine strain is thought to be in what was Mesopotamia, with strain lineages spread as grapevines were spread (Legras et al. 2007). Grapes were domesticated around the same time, suggesting that yeast domestication occurred simultaneously with that of grapes (Sicard and Legras 2011). Analyses of yeast genomic diversity of strains isolated from different technological sources are most consistent with independent domestication events having occurred for wine, beer, sake, and palm wine fermentations, with subsequent dispersion of those yeasts as the technology for production of these beverages spread from region to region (Sicard and Legras 2011). Fermentations represent very specific ecological niches that do not vary widely geographically, thus constraining the evolution of geographic diversity. However, human activities have clearly played a strong role in both the encouragement and limitation of wine strain diversity.

There is clear evidence in wine strain lineages of the imposition of evolutionary bottlenecks (Sicard and Legras 2011). An evolutionary bottleneck occurs when there is a strong selection against the majority of the strain diversity and in favor of one or a few traits in an ecological niche. In the case of wine strains, a bottleneck appears to have been introduced due to the use of sulfite as an antimicrobial agent during wine production (Peréz-Ortín et al. 2002, Yuasa et al. 2004). A rare chromosomal rearrangement occurred in one yeast lineage that resulted in the creation of a dominant allele of the sulfite pump $S S U 1-R$ that confers a high level of resistance to sulfite (Peréz-Ortín et al. 2002, Yuasa et al. 2004). Roughly $50 \%$ of the modern day wine isolates examined carry this specific chromosomal rearrangement (Yuasa et al. 2004). The widespread occurrence in nature of this specific chromosomal rearrangement suggests that the pervasive use of sulfite conferred such a growth advantage to strains carrying $S S U 1-R$ that the allele became fixed in the population. Similarly copper resistance is also predominant in wine strains, suggesting the use of copper in the vineyard strongly selected against strains that were copper sensitive (Kvitek et al. 2008).

Although analyses of genome structure suggest that wine strains are a highly related group when compared to the diversity of other members of the genus and species, there is significant phenotypic variation among wine strains. Interestingly, vineyard and winery strains do not segregate as separate clusters regardless of region of isolation, suggesting that these two populations mix freely. The discussion of whether yeast originates in the vineyard or the winery is moot at this point as there is too much exchange between these anthropic environments, likely mediated by insect transfer. It is also clear from these studies that the introduction of commercial strains will add to the phenotypic mix of the overall indigenous population and may provide genetic diversity that would not have arisen if these strains had not been introduced. The widespread penetration of the UCD522 genome across diverse wine-producing regions demonstrates that introduction of a genotype that is more fit for a technological environment will lead to spread of that genotype in the formation of mosaic lineages (Legras et al. 2007). Yeast strain diversity arises from a combination of genetic phenomena: spontaneous mutation and genetic drift, positive and negative trait selection imposed by ecological niche, inherent chromosomal instability, high rates of homologous recombination, and presence of nonhomologous chromosomal rearrangements.

\section{Genetic Mechanisms Underlying the Evolution of Genetic Diversity in Saccharomyces}

There are several genetic mechanisms by which genomes may change and phenotypic diversity emerge. Mutations or alterations of the coding sequence of the parental DNA can arise either due to DNA damage caused by external agents or more commonly by the oxidative intermediates of metabolism or errors in DNA replication or repair. Mutations may be beneficial, neutral, or detrimental to the cell depending upon its environment. Thus heterozygosity, the occurrence of allele diversity for a specific gene, happens naturally in yeast populations of individuals giving rise to genomic variation. Both sexual and asexual reproduction contribute to further population diversity. Recombination between chromosomes and loss of chromosome integrity can occur during asexual reproduction. Chromosomal repair mechanisms may introduce further genetic change or lead to the loss of one of a pair of alleles, termed gene conversion. Sexual reproduction by design leads to a reshuffling of genetic material across homologous chromosomes and also yields haploid progeny that will then self-mate (homodiploidization) or mate with a nonrelated haploid (heterodiploidization or outcrossing). Analysis of strain diversity suggests that reproduction in the wild is primarily asexual but that sexual reproduction occurs on average about every 1000 asexual generations (Warringer et al. 2011). However, the majority of sexual reproduction occurs between haploids derived from the same parent or from self-fertilization, with outcrossing a rarer event, one in every 50,000 divisions (Ruderfer et al. 2006, Tsai et al. 2008, Warringer et al. 2011).

Differences in chromosome number (aneuploidy) or numbers of chromosome sets (polyploidy) also occur as a consequence of natural selection and abortive cell cycles or aberrant cell fusion events. Genetic material can also be transferred across unrelated genera and species due to either cell fusion 
events (hybrid formation) or uptake and stabilization of DNA from the environment (lateral gene transfer). Analysis of wine strain diversity indicates that hybridization, the fusing of the genomes of two unrelated yeasts, as well as introgression, the incorporation of DNA from a different genus or species into the genome of the recipient, have occurred in the wild, creating strains that were more fit for localized vineyard or winery niches (Belloch et al. 2009, Dequin and Casaregola 2011, Gonzalez et al. 2007, 2008, Masneuf et al. 1998, Naumova et al. 2005).

Wine strains are typically homothallic diploids (Dequin and Casaregola 2011, Mortimer et al. 1994), meaning that they contain two sets of chromosomes and are able to undergo sporulation producing four haploid spores. The term homothallic refers to the trait of being self-fertile, which in the case of yeast means an isolated haploid cell has the ability to become diploid. Haploid spores may mate with each other following germination reproducing the diploid state. Alternately in homothallic strains, a haploid population displays switching of mating type, allowing mother and daughter cells to mate regenerating a diploid phase. Extensive crossing over between homologous chromosomes occurs during sporulation. The resolution of crossover regions leads to a reshuffling of the composition of the chromosomes. Recessive mutations can then be expressed in the haploid state, and if these mutations make the strain more fit for its environment, then they may confer a positive growth advantage. Thus, wine strains are able to undergo sporulation and quickly regenerate the diploid state from haploid progeny. The genomic composition of the spores will be the same as in the original parental strain but the spores will carry different arrangements of alleles that lead to the expression of different phenotypes. Any recessive mutations that arose during asexual or vegetative growth can then become expressed in the population and leads to the rapid evolution of diverse genotypes and ensures the survival of the species (Sipiczki 2008, Warringer et al. 2011). Cycles of sporulation and mating allow not only for the evolution of novel genomes with selective advantage in an ecological niche but also for the loss of detrimental alleles from the population, a process referred to as genome renewal (Mortimer et al. 1994).

Genetic differences or heterozygosities that lead to altered phenotypes are commonly thought of as being due to the appearance of a mutation in a coding sequence creating a protein of modified function or activity. Mutations in cis regulatory regions can also occur, changing the timing or level of production of a gene product, which may also lead to the appearance of a novel biological trait. These differences are referred to as single nucleotide polymorphisms, meaning that a single base pair in the DNA has changed. Many such mutations are neutral, that is, do not change either the primary sequence of a protein or its activity or regulation. However, changes in sequence that lead to a nonconservative change in the amino acid composition of the encoded protein may impact the functionality of the protein and lead to the appearance of an altered or new trait in the organism. In some cases these changes may be small insertions or deletions (indels) that alter the protein in some fundamental way or that may lead to loss of function altogether (Dowell et al. 2010). Such mutations arise naturally during DNA replication if a mismatch occurs that is not detected or correctly repaired. If the mutation occurs in a regulatory gene or complex, the impact can be profound and affect the expression of numerous genes. A lack of absolute fidelity in DNA replication ensures population genomic variation will occur and confers a rapid adaptive evolution capability to the population (Sipiczki 2008).

A change in phenotype of a cell may lead to selection for subsequent compensatory changes elsewhere in the genome (Sipiczki 2008, Warringer et al. 2011). The initiating mutation may lead to the evolution of subsequent changes that modify the impact of that mutation. These secondary mutations may enhance or suppress the original phenotype or modify its expression in the population. Specific trait variation shows a strong effect of population history (Warringer et al. 2011), meaning that the starting composition of a genome sets the stage for subsequent evolutionary and adaptive changes.

Alteration of gene copy number can also lead to the appearance of phenotypic diversity and drive the selection for compensatory mutation. Analysis of a set of deletion mutants of Saccharomyces showed that the appearance of aneuploidy was a common occurrence in such strains with entire chromosomes or parts of chromosomes amplified (Hughes et al. 2000). The loss of a gene conferring a selective disadvantage was compensated for by the amplification of a homolog of that gene from another chromosome (Hughes et al. 2000). These rare events conferred such a growth advantage that these cells eventually dominate the population. Even in diploid organisms loss of one allele can lead to haploinsufficiency, the situation in which the loss of one copy of a gene cannot be compensated for by the level of expression of the remaining copy, which can drive amplification of the unaltered copy of the gene (Deutschbauer et al. 2005). Such amplifications generally are not precise and result in amplification of a larger portion of the genome. Overexpression of some genes can also be deleterious in yeast and would lead to compensatory mutations. There is some debate in these situations as to whether the rate of mutation itself is increased by the biotic stress imposed by the initiator mutation or whether the domination by the modified population is simply due to the strong growth advantage that it confers to the cells.

In addition to this classical view of the origins of genetic diversity, other types of genome rearrangements can also occur that will modify gene expression and therefore lead to the development of altered phenotypes. Saccharomyces contains mobile repetitive elements called Ty elements that can hop around the genome (reviewed in Sipiczki 2011). Insertion of a Ty element into a gene may lead to a loss of function if a disruption of the coding sequence occurs. Insertion in a regulatory region can result in an altered expression profile for the gene, which can again lead to an altered phenotype. The presence of regions of homology created by the Ty elements can lead to recombination events across unrelated chromosomes, moving large regions of DNA from one chromosome to another (Sipiczki 2011). Significant diversity in the number 
and insertional positions of Ty elements has been observed in wine strains (Carreto et al. 2008).

Changes in the position and location of genes within chromosomes can arise via other mechanisms and also alter gene expression (Field et al. 2009, Tirosh et al. 2010). Chromosomal DNA is ordered in the nucleus by being woven around protein complexes called nucleosomes that are formed by histones and other chromatin-associated proteins (Jansen and Verstrepen 2011). The position of a gene with respect to nucleosome binding can affect access to RNA polymerase and therefore levels of expression (Lelandais et al. 2011).

The chromosomes of wine strains also show a diversity in size and on rare occasion in number (Bidenne et al. 1992, Briones et al. 1996, Carreto et al. 2008, Codon et al. 1998, Infante et al. 2003, Izquierdo Canas et al. 1997, Johnston et al. 2000, Vezinhet et al. 1990, Yamamoto et al. 1991). Chromosomes are attached to the nuclear membrane via telomeric regions and must deattach during replication as the centromeres become attached to the spindle pole bodies for segregation. On occasion this system fails and the tension on two anchored points of the same chromosome can result in chromosome breakage. Repair mechanisms save both ends of the chromosome but often they are not put back together precisely as they were before the break. This breakage and repair can lead to the significant chromosome or karyotype diversity that has been observed in Saccharomyces (Bidenne et al. 1992, Briones et al. 1996, Izquierdo Canas et al. 1997, Johnston and Mortimer 1986, Vezinhet et al. 1990, Yamamoto et al. 1991). The variation in karyotype in wine populations can be significant (Vezinhet et al. 1990, Yamamoto et al. 1991). In addition, given the dynamic nature of telomeric regions, genes located in these regions can be readily amplified during chromosome breakage and repair or due to normal recombination mechanisms. Once multiple copies of a gene exist, those individual copies may then diverge from each other, leading to the evolution of altered functions within the cell. The frequency of chromosomal rearrangements is strain dependent (Carro et al. 2003, Nadal et al. 1999). Multiple subpopulations with differing karyotypes may exist in the same fermentation (Longo and Vezinhet 1993, Miklos et al. 1997).

Entire sets of chromosomes or changes in cell ploidy also occur (Sipiczki 2011). Modern strains of Saccharomyces arose from an ancient genome duplication event that subsequently allowed divergence of duplicated genes to alternative or specialized functions (Kellis et al. 2004). The creation of two copies of a gene allows both to then undergo the process of natural mutation and selection and leads to diversification of cell functions. Increases in ploidy arise either as a failure of cell division or chromosomal segregation but can also arise due to abortive mating or cell fusion events within a species (Sicard and Legras 2011).

Hybrid yeast strains may be formed as a consequence of abortive attempts at sexual reproduction between different species of yeast. In this case, cell membranes fuse followed by fusion of the nuclei of the two organisms, creating an unstable number of chromosomes, some of which will then be lost (Ramirez et al. 2004). Chromosomal rearrangements also occur, leading to the formation of chimeric chromosomes. Several analyzed wine strains appear to have arisen from natural hybrid formation in nature (Belloch et al. 2009, Gonzalez et al. 2007, 2008, Masneuf et al. 1998, Naumova et al. 2005). In some cases hybridization occurred between haploid cells, in other cases diploid cells seem to have been involved or a haploid/diploid cell fusion event. Following hybridization chromosomal loss, rearrangements and chimeric chromosome formation may occur (Belloch et al. 2009) upon which natural selection will operate to enrich for the most-fit outcomes of the hybridization. The surviving populations of these events are stable mitotically, although often not able to engage in sexual reproduction with other strains.

In addition to genetic changes within a species, another source of genetic diversity can be the introduction of nonnative DNA from other organisms present in the population. This phenomenon is often referred to as lateral gene transfer and is observed in many population studies. In wine strains of Saccharomyces, introgression of DNA segments from unrelated organisms has also been observed (Novo et al. 2009). It is not clear that introgression arose due to lateral gene transfer, the pickup of DNA from the environment, rather than to the residual effects of abortive hybrid formation between unrelated yeasts. Sequence analysis of the commercial strain EC1118 revealed that this strain had undergone three independent introgression events and carries three large blocks of genetic information not typically found in other wine strains of Saccharomyces. These introgressions carried a total of 34 functional genes and have resulted in the expression of three relatively unique phenotypic traits (Dequin and Casaregola 2011, Novo et al. 2009).

Genetic changes may be neutral, beneficial, or detrimental. Neutral changes would confer no selective disadvantage in the immediate environment but may be useful as conditions change or may become detrimental. Beneficial genome changes confer some selective advantage to the current environment, generally leading to a dominance of that environment by that genotype. Detrimental genome changes can be severe and lead to cell death or may simply make cells less fit for the current environment, relegating those genotypes to a minor role. As with neutral and beneficial mutative changes, detrimental is likewise defined by the environment and what is detrimental under one condition may become beneficial under another. Often in fermentation multiple genotypes exist and the dominating genotype may change during fermentation as alternate genotypes become more favored. The development of a novel trait may be caused by a single mutation or recombination event or by the combinatorial effects of multiple mutations, a phenomenon referred to as quantitative, polygenic, or multilocus inheritance. Several complex genetic traits in wine strains of Saccharomyces are polygenic and it is a combination of alleles of divergent genes that leads to greater fitness (Bullard et al. 2010, Cubillos et al. 2011).

Genetic diversity arises from the operation of multiple mechanisms of DNA sequence divergence and genome alteration. The underlying processes are random events that 
are then selected according to environmental constraints. The ultimate selection is for the strain that is the most fit for the environment, that is, that can grow the fastest and attain the highest population density (Warringer et al. 2011). Modification of lag time in adaptation to a new environment seems less important of a selective factor than rate of growth and final cell biomass (Warringer et al. 2011). Subtle changes in an environment can tip the balance of fitness toward other genotypes present as subpopulations, allowing those populations to grow and displace the original dominant genotype. Genotypic change in wine yeast is a dynamic and recurrent process.

\section{Analytical Tools for Assessment of Yeast Diversity}

The genomic diversity of wine strains has been assessed in a variety of ways. There are two main features that are used to determine relatedness of genomes: sequence conservation, the differences in primary sequence of coding and noncoding regions, and gene synteny, the maintenance of the relative positioning of genes on a linear chromosome. Often differences in coding regions or in genes with highly conserved gene function may be weighted differently (to define genus) than in those regions or genes that are more free to evolve (to define species or strains). Although yeast do not possess the classic operon structure of bacteria, the colocalization of genes with related functions under the control of a single promoter, adjacencies of unrelated genes can be used to define strain relatedness. Rare recombination and crossover events can disrupt gene linkages. If the physical colocalization of genes shows similarity between strains, then those strains have not been separated evolutionarily long enough to demonstrate synteny divergence and are therefore related. Even if the primary sequence has diverged between two strains, a reflection of rates of spontaneous mutagenesis, selection, and adaptation, the conservation of the relative physical positions of the genes can be used to define the relationship of the two strains. Localized positioning also allows mapping of chromosomal rearrangements, the exchange of large regions of DNA between nonhomologous chromosomes, as the large region will maintain the gene positional orientation of the originating chromosome. Primary sequence divergence and loss of gene synteny are caused by different factors in evolution and occur at different rates in populations and thus provide complementary evidence in the delineation of strain lineages and relationships.

Two other types of evidence are also useful in determining strain relatedness, the number and position of mobile or repeated sequences, such as the yeast Ty elements and microsatellites, and assessment of the sequence of the mitochondrial genome of yeast (reviewed in Liti and Schacherer 2011). The mitochondrial DNA shows similar properties of primary sequence divergence and is inherited independently of the nuclear genome. The number and positioning of Ty elements can be used to define strain similarity, as even though these sequences are able to move within the genome such movement is rare enough that the positional location of these elements can define relatedness of the genomes under investigation (Ness et al. 2006, Rachidi et al. 1999). Microsatellites are regions of repetitive DNA also found in yeast. These regions can serve as sites of the initiation of homologous recombination among nonhomologous chromosomes and thus can be dispersed throughout the genome. Like Ty elements, the number, position, and conservation of sequence of microsatellite regions have diagnostic value in defining strain relatedness. Strain differences can also be compared using RiboPrinter technology to assess sequence diversity (Arvik et al. 2005).

Three comprehensive studies of yeast diversity used different methodologies. The first and broadest study used microsatellite typing of 651 strains (Legras et al. 2007). As this method is relatively easy to perform, a large collection of isolates can be processed. The clear relatedness of wine strains as a group independent of geographic origin or location (vineyard or winery) was demonstrated in this investigation (Legras et al. 2007). In a second study, oligonucleotide arrays were used to identify regions of sequence dissimilarity across a collection of 63 yeast strains (Schacherer et al. 2007, 2009). Oligonucleotide arrays consist of small roughly 10 to 20 mer oligos representing the genetic composition of the sequenced strain S288C (Gresham et al. 2006). If a mismatch occurs, meaning that a tested strain has a sequence that differs from that of the template reporter organism, the tested strain will not show hybridization to that spot. This method can be used to identify conserved and nonconserved regions across populations of organisms. However the absence of a signal could mean the loss of a gene or the retention of a modified version of that gene in the tested strain. Therefore, a subsequent analysis of strain diversity combined the use of these tiling arrays to identify divergent readings, followed by analysis of the region of the DNA via PCR amplification to determine if the absence of hybridization was due to deletion of that region of the chromosome or to areas of large sequence polymorphisms (Muller and McCusker 2011). In the majority of cases, the absence of signal was confirmed to be due to sequence divergence and not loss of the gene. These researchers were able to then examine the level of sequence divergence across physically colocalized genes to define large regions of sequence polymorphisms, that is, long stretches of DNA that were highly diverse between the tested and template strains. These large sequence polymorphisms were biased toward subtelomeric regions and also revealed the existence of introgressions in wine strains (Muller and McCusker 2011).

Another wide-ranging study of yeast diversity used a lowfold coverage of genome sequencing of over 70 strains to identify regions of differences (Liti et al. 2009). Direct sequencing provides a more comprehensive picture of genome variability but is also not biased by use of tiling arrays based on the $\mathrm{S} 288 \mathrm{C}$ genome. This analysis also led to the conclusion that wine strains are a more homologous group than would be expected, indicating that a single domestication event likely gave rise to the current diverse population of vineyard and winery isolates, consistent with previous analyses (Fay and 
Benavides 2005a). Finally, whole organism sequencing has been performed on several yeast strains (Argueso et al. 2009, Borneman et al. 2008, Liti et al. 2009, Novo et al. 2009, Wei et al. 2007). The availability of entire genomes provides the most robust assessment of relatedness and allowed identification of introgressions not only from other species of Saccharomyces but also from other yeast genera, providing a more complete depiction of the genomic constitution and population diversity of Saccharomyces.

The occurrence of linkage disequilibrium in wine strains has also been investigated (Schacherer et al. 2009). Linkage disequilibrium refers to the nonrandom association of alleles of distinct genes and can be thought of as an estimate of the conservation of mutated adjacent alleles within a chromosome. In other words, if a strain inherits allele A of gene A, how likely is it that it will also carry allele B of gene B versus another version of gene $\mathrm{B}$. This analysis showed that linkage disequilibrium was greater in clinical, distillery, and laboratory isolates than in wine isolates, indicating that the wine strain lineage is older than that of the other groups of strains (Schacherer et al. 2009).

Often, however, the mere existence of a sequence and cataloging of the genetic differences does not provide a complete picture of the phenotypic diversity of strains. Mutations may be neutral and some regions of the genome may tolerate variability without loss of function more than other regions, so other tools must be used to evaluate the impact of the genetic changes defined on the phenotype of the organism (Fay and Benavides 2005b). Neutral changes by definition reflect random genetic drift while those that confer a trait or phenotype can be selected for or against by the environment. The phenotype is the expression of the genotype and seemingly small changes in genotype may have a profound impact on phenotype. Two types of studies have been undertaken to define phenotypes in a comprehensive way to be compared to genotypic characterization. In one of these studies, a host of phenotypic traits, growth rates, growth requirements, resistances and sensitivities were used to characterize the relationship between genotype and trait variation (Warringer et al. 2011). Such massive screens are invaluable but timeconsuming to conduct.

Other researchers have relied on comparative transcript profiling (DeRisi et al. 1997, Kuthan et al. 2003, Lashkari et al. 1997, Lockhart et al 1996, Lockhart and Winzeler 2000, Schena et al. 1995, Velculescu et al. 1995) to infer phenotype from the pattern of expression of genes under defined conditions and to define the relationships among strains (Bullard et al. 2010, Cavalieri 2009, Kvitek et al. 2008, Lelandais et al. 2011, Rossouw et al. 2009, 2010). Conservation of a transcriptional response would indicate functional relatedness of the organisms under investigation (reviewed in Lelandais et al. 2011). The occurrence of two types of regulatory mutations have been investigated: those acting in trans, genes involved in signal generation, signal transduction, or regulated gene expression, and in cis, those occurring within the promoter or regulatory region of a gene or group of genes (Cavalieri et al. 2000, Cavalieri 2009). Changes in promoter regions that confer altered binding properties for regulatory factors can be found but do not appear to be drivers of diversity (Cavalieri 2009). In contrast, mutations that impact the binding capacity or regulatory functionality of trans factors have been identified in wild populations and do seem to underlie a component of strain diversity (Cavalieri 2009). A classic example of the impact of trans mutations in wine populations is the discovery of mutation of the SSY1 gene that is involved in sensing external amino acids (Brown et al. 2008, Cavalieri 2009). The loss of SSY1 results in cells unable to detect or respond to external amino acids and was identified in a vineyard population (Brown et al. 2008, Cavalieri et al. 2000). This single mutation impacted the expression of roughly 400 genes (Brown et al. 2008, Cavalieri et al. 2000). A change in a transcription factor can result in the appearance of multiple compensatory cis changes throughout the genome (Bullard et al. 2010). Proteomic and metabolite profiling has also been used to assess strain differences solely and in comparison to the transcriptome (Rossouw et al. 2008, 2009, 2010). These analyses indicate that, depending upon gene function, transcript profiles have predictive value for protein and metabolic activity. Further, changes in proteome and metabolome reflect changes in the genome and the population history of the strain and have value in defining strain relatedness in spite of the fact that a single mutation can have a broad impact on expression profiles.

\section{Functional Diversity of Wine Strains}

The analysis of wine strain diversity begins with the study of genomic relatedness within the genus Saccharomyces. There are currently eight recognized species within $S a c$ charomyces: S. arboricolus, S. bayanus, S. cariocanus, $S$. cerevisiae, S. kudriavzevii, S mikatae, S. paradoxus, and $S$. pastorianus (Kurtzman 2003, Kurtzman and Robnett 2003, Naumov 1996, Wang and Bai 2008). Saccharomyces pastoria$n u s$ is a hybrid species thought to have derived from a fusion event of S. cerevisiae and S. bayanus (reviewed in Dequin and Casaregola 2011). Analysis of trait variation suggests that S. cerevisiae, S. mikatae, and S. paradoxus form a cluster of strains with $S$. arboricolus, S. bayanus, and S. kudriavzevii forming a second cluster (Warringer et al. 2011). Saccharomyces pastorianus, S. cerevisiae, and S. bayanus are found associated with fermentations and anthropic environments and $S$. paradoxus and $S$. kudriavzevii have been found in vineyards. That these species inhabit similar ecological niches is confirmed by the discovery of interspecies hybrids. Wine isolates that are hybrids of $S$. cerevisiae and $S$. kudriavzevii, S. cerevisiae and S. bayanus, and a triple hybrid of S. cerevisiae, $S$. kudriavzevii, and S. bayanus have been identified in wine populations (Belloch et al. 2009, González et al. 2006, 2007, 2008, Lopandic et al. 2007, Masneuf et al. 1998, 2002). The majority of vineyard and winery isolates appear to be homothallic diploids and the main mode of cellular reproduction appears to be clonal rather than sexual (Legras et al. 2007). Roughly $28 \%$ of the over 600 wine and vineyard isolates examined were homozygous, suggesting that sporulation and self-diploidization occur in the wild (Legras et al. 2007). 
Two primary species of Saccharomyces are found during the alcoholic fermentation: $S$. cerevisiae and $S$. bayanus (formerly $S$. uvarum), with $S$. cerevisiae being the more prevalent (Sipiczki 2002). Occasionally, S. pastorianus can be found as can the hybrids of these yeasts (Naumov 1996). Sequence comparisons between $S$. cerevisiae and $S$. bayanus indicate $\sim 80 \%$ identity of coding sequences and $\sim 74 \%$ identity of noncoding sequences (Cliften et al. 2003).

Saccharomyces can be found in vineyards and winery environments. It is a common resident of winery flora and can be found in the vineyard albeit at a much lower frequency in the overall yeast flora population. Whether or not Saccharomyces is a true vineyard resident or just repeatedly introduced to the vineyard by winery operations has been much debated. Saccharomyces cerevisiae can be isolated from vineyards even in cases where the practice of placing spent yeast lees in the vineyard as fertilizer has not occurred (Clemente-Jimenez et al. 2004, Martini et al. 1996, Torok et al. 1996, Valero et al. 2005). Saccharomyces is commonly isolated from heavily damaged grapes (Mortimer and Polsinelli 1999) regardless of the nature of the damage. Mechanical damage to clusters leads to a bloom of yeast residents on the surface of the fruit and an increase in the population size of $S$. cerevisiae. Damage may also occur because of moldinduced cluster rot and Saccharomyces can be isolated from these environments as well. However, since rotting clusters attract insects and insects are vectors of Saccharomyces, it is not clear if the $S$. cerevisiae strains present arose from the surface of the fruit or not. Direct inoculation of vineyards with commercial yeasts did not lead to their establishment among the vineyard flora (Comitini and Ciani 2006, Valero et al. 2005), even when damaged berries were inoculated (Comitini and Ciani 2006). Since the level of inoculation in these studies was much higher than would be predicted from insect vectors, these data support the view that Saccharomyces can be considered a minor resident of the surface of a grape with conditions leading to the seepage of berry components selecting for Saccharomyces proliferation. With creation of fermentative conditions upon crushing of the fruit, even more strongly enriching conditions are presented to this microbe. Saccharomyces, initially present below detectable levels in spontaneous grape juice fermentations, will often be found as the dominate species at the end of fermentation, even under aseptic grape cluster harvesting conditions (Comitini and Ciani 2006, Valero et al. 2005). The population density of Saccharomyces in a winery is often higher than the density in the vineyard, and in the absence of selective pressure the greater number of winery yeast relative to vineyard yeast will lead to a greater contribution to the fermentation itself by the winery residents. In essence, Saccharomyces can be found in both the vineyard and the winery and which strains dominate a fermentation depend upon trait variation and selection imposed by the fermentation process.

Significant genetic diversity exists among wine strains of $S$. cerevisiae whether isolated from the vineyard or winery (Baleiras Couto et al. 1996, Briones et al. 1996, Gallego et al. 2005, Khan et al. 2000, Lopes et al. 2002, Sabate et al.
1998, Schuller et al. 2005, Schütz and Gafner 1993, 1994, Valero et al. 2006, Van der Westhuizen et al. 2000a, 2000b, Versavaud et al. 1995) and the same is true of $S$. bayanus (Sipiczki 2002). Analysis of over 1,600 isolates of S. cerevisiae from 54 spontaneous fermentations demonstrated the existence of 297 unique strains (Schuller et al. 2005). In a more limited study, 13 out of 16 isolates $(81 \%)$ were determined to be unique strains and the four identical strains were isolated from the same location (Baleiras Couto et al. 1996). Even higher ratios of unique genotypes have been found- $87.5 \%$ (Valero et al. 2006), 81 to $91 \%$ (Gallego et al. 2005), and 91 to $96 \%$ (Schuller et al. 2005) - depending on the technique used. The highest numbers of unique genotypes in all of these studies are represented by a single isolate, indicating that the true extent of the diversity present in the wild is still being underestimated. Significant strain diversity exists within the same vineyard environment, suggesting the importance of localized conditions for the selection of genetically modified strains or, alternately, the existence of factors driving genetic change. One such factor may be exposure to ultraviolet light. Metabolites produced either by the plant or by other microbes in the environment, such as mycotoxins, may also serve to accelerate the appearance of genetic differences in the absence of any direct selective pressure. Interestingly in studies with large enough populations of both vineyard and winery isolates, strains do not cluster genomically based upon site of origin (Legras et al. 2007), again indicating that there is significant flux and comingling between the vineyard and winery populations.

Not surprisingly, the fitness of strains for specific fermentation niches also has been found to vary. In some cases, only one or a few strains dominate throughout fermentation (Versavaud et al. 1995). In contrast, other researchers have found that different strains dominate at different stages of the fermentation (Egli et al. 1998, Sabate et al. 1998, Schütz and Gafner 1993) or that several strains of Saccharomyces appear to be simultaneously present in equivalently high numbers (Torija et al. 2001, Vezinhet et al. 1992). Presumably, the biodiversity of wine strains in the environment results in these different patterns of dominance in fermentations. Strains that are dominant in one environment may not show the same degree of dominance in another because the strain attributes conferring dominance may be best suited to the fermentation conditions of a specific winery or vintage. As conditions of production change, different strains may become dominant. Assessment of strain diversity across vintages has shown that different strains are present each year (Gutierrez et al. 1999, Schuller et al. 2005).

The genetic diversity of wine yeasts has also been documented using genomic sequence comparisons and functional genomic analysis of transcript profiles (Borneman et al. 2008, Dunn et al. 2005, Fay et al. 2004, Gresham et al. 2006, Legras et al. 2007, Liti et al. 2006, 2009, Schacherer et al. 2009, Townsend et al. 2003, Tsai et al. 2008, Winzeler et al. 2003). Strains that are undistinguishable from each other by genomic or mitochondrial DNA profiling may carry mutations leading to changes in important enological phenotypes, particularly 
if the genetic differences are targeted to high-impact genes (such as transcription factors) or genes involved in flavor modification or production. Indeed, analyses of the presence of single nucleotide polymorphisms (SNP) suggest that they exist across populations of Saccharomyces with a frequency of 2.8 SNPs per kilobase of DNA (Schacherer et al. 2009). In a sequence comparison of wine strain AWRI1631 to S288c, a SNP frequency of 1 per 150 base pairs, or roughly 7 SNPs per kilobase, was found (Borneman et al. 2008). SNPs occur less frequently in genes located near the centromere and more frequently for genes located in subtelomeric regions (Schacherer et al. 2009). Deletions of genetic material also occur (Schacherer et al. 2009) but are found at a very low frequency in the essential genes. Thus, there is the potential for significant variation in gene expression profiles as a consequence of underlying genetic differences across strains, making comparisons of strains grown under different conditions challenging.

The sequence of a commercial isolate of EC1118 revealed that this strain contains large stretches of DNA not normally found in wine yeast and not thought to originate from $S$. cerevisiae (Novo et al. 2009). If genetically modified organism (GMO) is defined as the presence of nonnative DNA, then EC1118 would be considered to be a naturally arising GMO. The genes of these regions contained by EC1118 carry functional genes that impact the fermentation phenotypes of this strain. EC1118 possesses an analog of the FSY1 gene also found in $S$. pastorianus that encodes a fructose proton symporter (Galeote et al. 2010). If functional as such in EC118, this transporter would couple fructose movements to those of protons and lead to more efficient transport of fructose into the cell. This transporter has a high affinity for fructose in contrast to the native $H X T$ hexose transporter genes and is thought to be responsible for the elevated use of fructose late in fermentation seen in EC1118. EC1118 also carries a gene encoding a protein similar to the peptide transporters found in fungi and can therefore perhaps transport a wider array of peptides to be broken down internally and used as nitrogen source (Damon et al. 2011). The source of this introgression is unknown. The third introgression carries genes with close relatives in Zygosaccharomyces bailii (Novo et al. 2009), suggesting it may have arisen from this yeast. This particular region is not unique to EC1118 and is found in other wine yeast strains (Dequin and Casaregola 2011, Galeote et al. 2011). This region contains an autonomously replicating sequence, suggesting that it may be able to amplify in the genome. In support of the amplification hypothesis, some wine strains have been found to carry multiple regions of this DNA (Galeote et al. 2011). The function(s) of this region are not yet known, but its appearance in many wine strains and amplification therein suggest a positive selection for the presence of this region in wine strains. Some of the winemaking traits that have made EC1118 a popular selection for wine production may have indeed arisen from natural introgression from other species and genera of yeast.

Genomic analyses have revealed that many commercial strains have acquired altered signaling properties (Verstrepen et al. 2004) and these signaling differences may be important for differential tolerance to various stressors. It is likely that increased basal levels of expression of genes involved in tolerance to stressful conditions allows more rapid adaptation to those conditions and, therefore, enhance survival (Bisson et al. 2007). However, high basal levels of expression of these genes may result in slower initial growth rates and lack of an ability to dominate fermentations (Bisson et al. 2007). There appears to be a dynamic interplay between expression of genes associated with stress tolerance and those associated with rapid growth (Bisson et al. 2007). Given the existence of multiple stressors in the environment and the feast or famine atmosphere of growth on the surface of fruits, it is not surprising that vast biodiversity of both genetic composition and gene expression profiles is observed in native Saccharomyces isolates.

Commercial and native yeast isolates display greater genomic and genetic instability than laboratory strains (Ambrona et al. 2005), and aberrations in the number of some chromosomes are common (Bakalinsky and Snow 1990). Wild strains are generally homothallic and show low sporulation rates and poor spore viability. They also display a high degree of heterozygositiy, chromosomal polymorphisms and rearrangements, and karyotype instability (Carro and Pina 2001, Codon et al. 1998, Hughes et al. 2000, Izquierdo Canas et al. 1997, Johnston et al. 2000, Landry et al. 2006a, 2006b, Longo and Vezinhet 1993, Mortimer 2000, Myers et al. 2004, Oshiro and Winzeler 2000). The dynamic nature of the genome likely poses a distinct advantage in the environment, as evidenced by the extensive diversity observed among native isolates from the same site (Hauser et al. 2001). The biodiversity of wine strains of Saccharomyces is likely a consequence of both natural selection and random mutagenesis and accumulation of mutations. Wild yeasts show elevated rates of spontaneous mutagenesis which, if followed by sporulation and diploidization, can lead to the rapid creation of significant diversity across a population. The return to a homozygous state has been termed "genome renewal" (Ambrona and Ramierez 2007, Mortimer et al. 1994). Some underlying features of gene expression in wine strains will likely be conserved across this rich biodiversity while others may show striking strain dependence. It is important to note that comparisons of gene expression in recently isolated native strains of $S$. cerevisiae versus those that have been cultivated in laboratories demonstrate clear differences in expression profiles of wild strains and their domesticated derivatives (Kuthan et al. 2003, Palkova 2004). Strains rapidly lose some phenotypes associated with growth in the wild upon laboratory cultivation (Palkova 2004).

\section{Impact of Strain Diversity on Wine Production}

Even though constrained by the demands of a highly specialized ecological niche, grape juice fermentation, significant diversity exists among wine isolates of Saccharomyces. That no one strain represents the entire spectrum of wine yeast diversity is both beneficial and problematic for wine production. The beneficial impact of such diversity is the ability to 
obtain different flavor and aroma profiles of the wine depending upon which strain has been used. Of course, astute use of diversity in this manner means understanding the underlying genetic capabilities of the yeast strains themselves as well as the potential for flavor and aroma evolution due to the composition of the grapes at harvest. The challenge of diversity for wine production is that strain nutritional needs, tolerances of stresses such as temperature and ethanol, compatibility with other microbes present, and fermentation characteristics will vary such that one recipe will not apply to all strains.

Fermentation conditions may also foster the development of diversity in the population within the fermentation. Whether one strain dominates throughout a fermentation or there is a progressive change in dominant genotypes depends upon the starting genomic composition and phenotypic constitution of the initiator populations, their relative numbers, and the selective potential imposed by fermentation conditions. Temperature has been shown to be an important driver of strain evolution (Salvado et al. 2011). Temperatures of grape processing, must or juice holding, and of fermentation itself pose selections for specific types of strains. Use of nutritional supplements also impacts strain diversity and persistence. Strains carrying the ssyl mutation (Brown et al. 2008) clearly detect nitrogen in the fermentation differently than strains not mutated in this gene. The widespread use of sulfite has also imposed a selection in favor of sulfite-resistant genotypes. Thus, the imposition of specific fermentation conditions can directly influence population diversity and persistence.

The analysis of a large collection of strains by Legras et al. (2007) illustrates another important facet of wine strain diversity. The discovery of genetic signatures of the UCD522 strain in diverse populations of winery and vineyard yeasts isolated from around the world demonstrates the penetrance and persistence a niche-favored genome can have in wineproducing regions. It further demonstrates that use of commercial strains may impact the genetic composition of the native populations through outcrossing or hybrid formation followed by selection for fitness. The genomes of fermentation strains are in a constant state of flux and evolution. The process of fermentation is thought to impose a strong selective pressure and therefore is a powerful evolutionary force in the generation of diversity (Cubillos et al. 2011, Kvitek et al. 2008, Sipiczki 2011, Spor et al. 2009, Warringer et al. 2011).

Perhaps the most surprising conclusion from the analysis of wine strain diversity is the existence of interspecies hybrids (Belloch et al. 2009, González et al. 2006, 2007, 2008, Lopandic et al. 2007, Masneuf et al. 1998, 2002) and introgressions from non-Saccharomyces genera (Damon et al. 2011, Galeote et al. 2010, Novo et al. 2009). These variants have arisen naturally in native populations and demonstrate the potential for development of novel reproductively isolated organisms. Hybrid genomes appear to be persistent in the wild despite greater reproductive isolation and such hybrids confer a fitness advantage since they seem to have evolved independently. Hybrids display complex patterns of gene expression (Tirosh et al. 2010) and set the stage for rapid evolution of further diversity.

\section{Conclusions}

Extensive analyses of strain variation within $S$. cerevisiae have provided a wealth of information on the origin of wine strains and the subsequent evolution of strain diversity. Several studies have independently confirmed the view of a single domestication event having given rise to the modern population of vineyard and winery isolates with the spread of wine strains paralleling the migration of grapevines. There is simultaneously greater genomic similarity and greater phenotypic variation in the wine strains analyzed. The genomic similarity suggests the presence of evolutionary bottlenecks, the selection for fewer genotypes in a population, with subsequent reamplification of diversity. Sulfite use appears to be one such bottleneck imposed in the Middle Ages. Diversity arises from the well-described processes of spontaneous mutagenesis, genetic drift, and environmental selection. The knowledge gained from the analysis of wine strain diversity and the correlation between genotype and phenotype can be used in the development and identification of specific strains well-tailored for specific production needs.

\section{Literature Cited}

Ambrona, J., and M. Ramirez. 2007. Analysis of homothallic Saccharomyces cerevisiae strain mating during must fermentation. Appl. Environ. Microbiol. 73:2486-2490.

Ambrona, J., A. Vinagre, and M. Ramirez. 2005. Rapid asymmetrical evolution of Saccharomyces cerevisiae wine yeasts. Yeast 22:12991306.

Argueso, J.L., et al. 2009. Genome structure of a Saccharomyces strain widely used in bioethanol production. Genome Res. 19:2258-2270.

Arvik, T., T. Henick-Kling, and J. Gafner. 2005. Automated genotyping of Saccharomyces cerevisiae using RiboPrinter. Int. J. Food Microbiol. 104:35-41.

Bakalinsky, A.T., and R. Snow. 1990. The chromosomal constitution of wine strains of Saccharomyces cerevisiae. Yeast 6:367-382.

Baleiras Couto, M.M., B. Eijsma, H. Hofstra, J.H.J Huis in’t Veld, and J.M.B.M van der Vossen. 1996. Evaluation of molecular typing techniques to assign genetic diversity among Saccharomyces cerevisiae strains. Appl. Environ. Microbiol. 62:41-46.

Belloch, C., R. Perez-Torrado, S.S. Gonzalez, J.E. Perez-Ortin, J. Garcia-Martinez, A. Querol, and E. Barrio. 2009. Chimeric genomes of natural hybrids of Saccharomyces cerevisiae and Saccharomyces kudriavzevii. Appl. Environ. Microbiol. 75:2534-2544.

Bidenne, C., B. Blondin, S. Dequin, and F. Vezinhet. 1992. Analysis of the chromosomal DNA polymorphism of wine strains of Saccharomyces cerevisiae. Curr. Genet. 22:1-7.

Bisson, L.F., J.E. Karpel, V. Ramakrishnan, and L. Joseph. 2007. Functional genomics of wine yeast Saccharomyces cerevisiae. Adv. Food Nutr. Res. 53:65-121.

Borneman, A.R., A.H. Forgan, I.S. Pretorius, and P.J. Chambers. 2008. Comparative genome analysis of a Saccharomyces cerevisiae wine strain. FEMS Yeast Res. 8:1185-1195.

Briones, A.I., J. Ubeda, and M.S. Grando. 1996. Differentiation of Saccharomyces cerevisiae strains isolated from fermenting musts according to karyotype patterns. Int. J. Food Microbiol. 28:369-377.

Brown, K.M., C.R. Landry, D.L. Hartl, and D. Cavalieri. 2008. Cascading transcriptional effects of a naturally occurring frameshift mutation in Saccharomyces cerevisiae. Mol. Ecol. 17:2985-2997.

Bullard, J.H., Y. Mostovoy, S. Dudoit, and R.B. Brem. 2010. Polygenic and directional regulatory evolution across pathways in Saccharomyces. Proc. Natl. Acad. Sci. USA 107:5058-5063. 
Carreto, L., M.F. Eiriz, A.C. Gomes, P.M. Pereira, D. Schuller, and M.A.S. Santos. 2008. Comparative genomics of wild type strains unveils important genomic diversity. BMC Genomics 9:524.

Carro, D., and B. Pina. 2001. Genetic analysis of the karyotype instability in natural wine yeast strains. Yeast 18:1457-1470.

Carro, D., E. Bartra, and B. Pina. 2003. Karyotype rearrangements in a wine yeast strain by rad52-dependent and rad52-independent mechanisms. Appl. Environ. Microbiol. 69:2161-2165.

Cavalieri, D. 2009. Evolution of transcriptional regulatory networks in yeast populations. Syst. Biol. Med. 2:324-335.

Cavalieri, D., J.P. Townsend, and D.L. Hartl. 2000. Manifold anomalies in gene expression in a vineyard isolate of Saccharomyces cerevisiae revealed by DNA microarray analysis. Proc. Natl. Acad. Sci. USA 97:12369-12374

Clemente-Jimenez, J.M., L. Mingorance-Carzola, S. MartinezRodriguez, F.J. Las Heras-Vazquez, and F. Rodriguez-Vico. 2004. Molecular characterization and oenological properties of wine yeasts isolated during spontaneous fermentation of six varieties of grape must. Food Microbiol. 21:149-155.

Cliften, P., P. Sudarsanam, A. Desikan, L. Fulton, B. Fulton, J. Majors, R. Waterston, B.A. Cohen, and M. Johnston. 2003. Finding functional features in Saccharomyces genomes by phylogenetic footprinting. Science 301:71-76.

Codon, A.C., T. Benitez, and M. Korhola. 1998. Chromosomal polymorphism and adaptation to specific industrial environments of Saccharomyces strains. Appl. Microbiol. Biotech. 49:154-163.

Comitini, F., and M. Ciani. 2006. Survival of inoculated Saccharomyces cerevisiae strain on wine grapes during two vintages. Lett. Appl. Microbiol. 42:248-253.

Cubillos, F.A., E. Billi, E. Zorgo, L. Parts, P. Fargier, S. Omholt, A. Blomberg, J. Warringer, E.J. Louis, and G. Liti. 2011. Assessing the complex architecture of polygenic traits in diverse yeast populations. Mol. Ecol. 20:1401-1413.

Damon, C., L. Vallon, S. Zimmermann, M.Z. Haider, V. Galeote, S Dequin, P. Luis, L. Fraissinet-Tachet, and R. Marmeisse. 2011. A novel fungal family of oligopeptide transporters identified by functional metatranscriptomics of soil eukaryotes. ISME J. 5:1871-1880.

Dequin, S., and S. Casaregola. 2011. The genomes of fermentative Saccharomyces. C.R. Biologies 334:687-693.

DeRisi, J.L., V.R. Iyer, and P.O. Brown. 1997. Exploring the metabolic and genetic control of gene expression on a genomic scale. Science 278:680-686

Deutschbauer, A.M., D.F. Jaramillo, M. Proctor, J. Kumm, M.E. Hillenmeyer, R.W. Davis, C. Nislow, and G. Giaever. 2005. Mechanisms of haploinsufficiency revealed by genome-wide profiling in yeast. Genetics 169:1915-1925.

Diezmann, S., and F.S. Dietrich. 2009. Saccharomyces cerevisiae: Population divergence and resistance to oxidative stress in clinical, domesticated and wild isolates. PLoS ONE 4(4):e5317.

Dowell, R.D., et al. 2010. Genotype to phenotype: A complex problem. Science 328:469.

Dunn, B., R.P. Levine, and G. Sherlock. 2005. Microarray karyotyping of commercial wine yeast strains reveals shared, as well as unique, genomic signatures. BMC Genomics 6:1-21.

Egli, C.M., W.D. Edinger, C.M. Mitrakul, and T. Henick-Kling. 1998. Dynamics of indigenous and inoculated yeast populations and their effect on the sensory character of Riesling and Chardonnay wines. J. Appl. Microbiol. 85:779-789.

Ezeronye, O.U., and J.L. Legras. 2009. Genetic analysis of Saccharomyces cerevisiae strains isolated from palm wine in eastern Nigeria. Comparison with other African strains. J. Appl. Microbiol. 106:1569-1578.

Fay, J.C., and J.A. Benavides. 2005a. Evidence for domesticated and wild populations of Saccharomyces cerevisiae. PLoS Genet. 1(1):e5.
Fay, J.C., and J.A. Benavides. 2005b. Hypervariable noncoding sequences in Saccharomyces cerevisiae. Genetics 170:1575-1587.

Fay, J.C., H.L. McCullough, P.D. Sniegowski, and M.B. Eisen. 2004. Population genetic variation in gene expression is associated with phenotypic variation in Saccharomyces cerevisiae. Genome Biol. 5:R26.

Field, Y., Y. Fondufe-Mittendorf, I. Moore, P. Mieczkowski, N. Kaplan, Y. Lubling, J. Lieb, J. Widom, and E. Segal. 2009. Gene expression divergence in yeast is coupled to evolution of DNA-encoded nucleosome organization. Nat. Genet. 41:438-445.

Galeote, V., M. Novo, M. Salema-Oom, E. Brion, E. Valerio, P. Goncalves, and S. Dequin. 2010. FSY1, an horizontally transferred gene in the Saccharomyces cerevisiae EC1118 wine yeast strain encodes a high affinity fructose $/ \mathrm{H}^{+}$symporter. Microbiology 156:3754-3761.

Galeote, V., F. Bigey, E. Beyne, M. Novo, J.L. Legras, S. Casaregola, and S. Dequin. 2011. Amplification of a Zygosaccharomyces bailii DNA segment in wine yeast genomes by extrachromosomal circular DNA formation. PLoS ONE 6(3):e17872.

Gallego, F.J., M.A. Perez, Y. Nunez, and P. Hildago. 2005. Comparison of RAPDs, AFLPs and SSR marker for genetic analysis of yeast strains of Saccharomyces cerevisiae. Food Microbiol. 22:561-568.

González, S.S., E. Barrio, J. Gafner, and A. Querol. 2006. Natural hybrids from Saccharomyces cerevisiae, Saccharomyces bayanus and Saccharomyces kudriavzevii in wine fermentation. FEMS Yeast Res. 6:1221-1234.

González, S.S., L. Gallo, M.A. Climent, E. Barrio, and A. Querol. 2007. Enological characterization of natural hybrids from Saccharomyces cerevisiae and S. kudriavzevii. Int. J. Food Microbiol. 116:11-18.

González, S.S., E. Barrio, and A. Querol. 2008. Molecular characterization of new natural hybrids of Saccharomyces cerevisiae and S. kudriavzevii in brewing. Appl. Envion. Microbiol. 74:2314-2320.

Gresham, D., D.M. Ruderfer, S.C. Pratt, J. Schacherer, M.J. Dunham, D. Botstein, and L. Kruglyak. 2006. Genome-wide detection of polymorphisms at nucleotide resolution with a single DNA microarray. Science 311:1932-1936

Gutierrez, A.R., P. Santamaria, S. Epifanio, P. Garijo, and R. Lopez. 1999. Ecology of spontaneous fermentation in one winery during 5 consecutive years. Lett. Appl. Microbiol. 29:411-415.

Hauser, N.C., K. Fellenberg, R. Gil, S. Bastuck, J.D. Hoheisel, and J.E. Perez-Ortin. 2001. Whole genomes of a wine yeast strain. Comp. Funct. Genomics 2:69-79.

Hughes, T.R., et al. 2000. Widespread aneuploidy revealed by DNA microarray expression profiling. Nat. Genet. 25:333-337.

Infante, J.J., K.M. Dombek, L. Rebordinos, J.M. Cantoral, and E.T. Young. 2003. Genome-wide amplifications caused by chromosomal rearrangements play a major role in the adaptive evolution of natural yeast. Genetics 165:1745-1759.

Izquierdo Canas, P.M., J.F. Ubeda Iranzo, and A.I. Briones Perez. 1997. Study of the karyotype of wine yeasts isolated in the region of Valdepenas in two consecutive vintages. Food Microbiol. 14:221-225.

Jansen, A., and K.J. Verstrepen. 2011. Nucleosome positioning in Saccharomyces cerevisiae. Microbiol. Molec. Biol. Rev. 75:301-320.

Johnston, J.R., and R.K. Mortimer. 1986. Electrophoretic karyotyping of laboratory and commercial strains of Saccharomyces and other yeasts. Int. J. Syst. Bacteriol. 4:569-572.

Johnston, J.R., C. Baccari, and R.K. Mortimer. 2000. Genotypic characterization of strains of commercial wine yeasts by tetrad analysis. Res. Microbiol. 151:583-590.

Kellis, M., B.W. Birren, and E.S. Lander. 2004. Proof and evolutionary analysis of ancient genome duplication in the yeast Saccharomyces cerevisiae. Nature 428:617-624.

Khan, W., O.P.H. Augustyn, T.J. Van der Westhuizen, M.G. Lambrechts, and I.S.Pretorius. 2000. Geographic distribution and evaluation of Saccharomyces cerevisiae strains isolated from vineyards in the 
warmer inland regions of the Western Cape in South Africa. S. Afr. J. Enol. Vitic. 21:17-31.

Kurtzman, C.P. 2003. Phylogenetic circumscription of Saccharomyces, Kluyveromyces and other members of the Saccharomycetaceae, and the proposal of the new genera Lachancea, Nakaseomyces, Naumovia, Vanderwaltozyma and Zygotorulaspora. FEMS Yeast Res. 4:233-245.

Kurtzman, C.P., and C.J. Robnett. 2003. Phylogenetic relationships among yeasts of the 'Saccharomyces complex' determined from multigene sequence analysis. FEMS Yeast Res. 3:417-432.

Kuthan, M., F. Devaux, B. Janderova, I. Slaninova, C. Jacq, and Z. Palkova. 2003. Domestication of wild Saccharomyces cerevisiae is accompanied by changes in gene expression and colony morphology. Mol. Microbiol. 47:745-754.

Kvitek, D.J., J.L. Will, and A.P. Gasch. 2008. Variation in stress sensitivity and genomic expression in diverse $S$. cerevisiae isolates. PLoS Genet. 4(10):e1000223.

Landry, C.R., J. Oh, D.L. Hartl, and D. Cavalieri. 2006a. Genomewide scan reveals that genetic variation for transcriptional plasticity in yeast is biased towards multi-copy and dispensable genes. Gene 366:343-351.

Landry, C.R., J.P. Townsend, D.L. Hartl, and D. Cavalieri. 2006 b. Ecological and evolutionary genomics of Saccharomyces cerevisiae. Mol. Ecol. 15:575-591.

Lashkari, D.A., J.L. DeRisi, J.H. McCusker, A.F. Namath, C. Gentile, S.Y. Hwang, P.O. Brown, and R.W. Davis. 1997. Yeast microarrays for genome wide parallel genetic and gene expression analysis. Proc. Natl. Acad. Sci. USA 94:13057-13062.

Legras, J.L., D. Merdinoglu, J.M. Cornuet, and F. Karst. 2007. Bread, beer and wine: Saccharomyces cerevisiae diversity reflects human history. Mol. Ecol. 16:2091-2102.

Lelandais, G., C. Goudot, and F. Devaux. 2011. The evolution of gene expression regulatory networks in yeasts. C.R. Biologies 334(89):655-661

Liti, G., and J. Schacherer. 2011. The rise of yeast population genomics, C.R. Biologies 334(8-9):612-619.

Liti, G., D.B.H. Barton, and E.J. Louis. 2006. Sequence diversity, reproductive isolation and species concepts in Saccharomyces. Genetics 174:839-850.

Liti, G., et al. 2009. Population genomics of domestic and wild yeast. Nature 458:337-341.

Lockhart, D.J., and E.A. Winzeler. 2000. Genomics, gene expression and DNA arrays. Nature 405:827-836.

Lockhart, D.J., et al. 1996. Expression monitoring by hybridization to high-density oligonucleotide arrays. Nat. Biotechnol. 14:1675-1680.

Longo, E., and F. Vezinhet. 1993. Chromosomal rearrangements during vegetative growth of a wild strain of Saccharomyces cerevisiae. Appl. Environ. Microbiol. 59:322-326.

Lopandic, K., H. Gangl, E. Wallner, G. Tscheik, G. Leitner, A. Querol, N. Borthe, M. Breitenbach, H. Prillinger, and W. Tiefenbrunner. 2007. Genetically different wine yeasts isolated from Austrian vinegrowing regions influence wine aroma differently and contain putative hybrids between Saccharomyces cerevisiae and Saccharomyces kudriavzevii. FEMS Yeast Res. 7:953-965.

Lopes, C.A., M. van Broock, A. Querol, and A.C. Caballero. 2002. Saccharomyces cerevisiae wine yeast populations in a cold region in Argentinean Patagonia. A study at different fermentation scales. J. Appl. Microbiol. 93:608-615.

Martini, A., M. Ciani, and G. Scorzetti. 1996. Direct enumeration and isolation of wine yeasts from grape surfaces. Am. J. Enol. Vitic. 47:435-440.

Masneuf, I., J. Hansen, C. Groth, J. Piskur, and D. Dubourdieu. 1998. New hybrids between Saccharomyces sensu stricto yeast species found among wine and cider production strains. Appl. Environ. Microbiol. 64:3887-3892.
Masneuf, I., M.L. Murat, G.I. Naumov, T. Tominaga, and D. Dubourdieu. 2002. Hybrids of Saccharomyces cerevisiae x Saccharomyces bayanus var. uvarum having a high liberating ability of some sulfur varietal aromas of Vitis vinifera Sauvignon blanc wines. J. Int. Sci. Vigne Vin 36:205-212.

McGovern, P.E., D.L. Glusker, L.J. Exner, and M.M. Voight. 1996. Neolithic resonated wine. Nature 381:480-481.

Miklos, I., T. Varga, A. Nagy, and M. Sipiczki. 1997. Genome instability and chromosomal rearrangements in a heterothallic wine yeast. J. Basic Microbiol. 37:345-354.

Mortimer, R.K. 2000. Evolution and variation of the yeast (Saccharomyces) genome. Genome Res. 10:403-409.

Mortimer, R.K., and J.R. Johnston. 1986. Genealogy of principal strains of the yeast genetic stock center. Genetics 113:35-43.

Mortimer, R., and M. Polsinelli. 1999. On the origin of wine yeast. Res. Microbiol. 150:199-204.

Mortimer, R.K., P. Romano, G. Suzzi, and M. Polsinelli. 1994. Genome renewal: A new phenomenon revealed from a genetic study of 43 strains of Saccharomyces cerevisiae derived from natural fermentation of grape musts. Yeast 10:1543-1552.

Muller, L.A., and J.H. McCusker. 2011. Nature and distribution of large sequence polymorphisms in Saccharomyces cerevisiae. FEMS Yeast Res. 11(7):587-594.

Myers, C.L., M.J. Dunham, S.Y. Kung, and O.G. Troyanskaya. 2004. Accurate detection of aneuploidies in array CGH and gene expression microarray data. Bioinformatics 20:3533-3543.

Nadal, D., D. Carro, L.J. Fernandez, and B. Pina. 1999. Analysis and dynamics of the chromosomal complement of wild sparkling-wine yeast strains. Appl. Environ. Microbiol. 65:1688-1695.

Naumov, G. 1996. Genetic identification of biological species in the Saccharomyces sensu stricto complex. J. Ind. Microbiol. Biotechn. 17:295-302.

Naumova, E.S., G.I. Naumov, I. Masneuf-Pomarede, M. Aigle, and D. Dubourdieu. 2005. Molecular genetic study of introgression between Saccharomyces bayanus and S. cerevisiae. Yeast 22:1099-1115.

Ness, F., F. Lavailée, D. Dubourdieu, M. Agile, and L. Dulau. 2006. Identification of yeast strains using the polymerase chain reaction. J. Sci. Food Agric. 62:89-94.

Novo, M., et al. 2009. Eukaryote-to-eukaryote gene transfer events revealed by the genome sequence of the wine yeast Saccharomyces cerevisiae EC1118. Proc. Natl. Acad. Sci. USA 106:16333-16338.

Oliver, S.G., M.K. Winson, D.B. Kell, and F. Baganz. 1998. Systematic functional analysis of the yeast genome. Trends Biotechnol. 16:373-378.

Oshiro, G., and E.A. Winzeler. 2000. Aneuploidy_it's more common than you think. Nat. Biotech. 18:715-716.

Palkova, Z. 2004. Multicellular microorganisms: Laboratory versus nature. EMBO Reports 5:470-476.

Pasteur, L. 1860. Memorie sur la fermentation alcoolique. Ann. Chim. Phys. 58:323-426.

Peréz-Ortín, J.E., A. Querol, S. Puig, and E. Barrio. 2002. Molecular characterization of a chromosomal rearrangement involved in the adaptive evolution of yeast strains. Genome Res. 12:1533-1539.

Rachidi, N., P. Barre, and B. Blondin. 1999. Multiple Ty-mediated chromosomal translocations lead to karyotype changes in a wine strain of Saccharomyces cerevisiae. Mol. Gen. Genet. 261:841-850.

Ramirez, M., A. Vinagre, J. Ambrona, F. Molina, M. Maqueda, and J.E. Robello. 2004. Genetic instability of heterozygous, hybrid, natural wine yeasts. Appl. Environ. Microbiol. 74:2129-2143.

Roberts, I.N., and S.G. Oliver. 2010. The yin and yang of yeast: Biodiversity research and systems biology as complementary forces driving innovation in biotechnology. Biotech. Letts. 33:477-487. 
Rossouw, D., T. Naes, and F. Bauer. 2008. Linking gene regulation and the exo-metabolome: A comparative transcriptomics approach to identify genes that impact the production of volatile aroma compounds in yeast. BMC Genomics 9:530.

Rossouw, D., R. Olivares-Hernandes, J. Nielsen, and F.F. Bauer. 2009. Comparative transcriptome approach to investigate differences in wine yeast physiology and metabolism during fermentation. Appl. Environ. Microbiol. 75:6600-6612.

Rossouw, D., A.H. van den Dool, D. Jacobson, and F.F. Bauer. 2010, Comparative transcriptome and proteomic profiling of industrial wine yeast strains. Appl. Environ. Microbiol. 76:3911-3923.

Ruderfer, D.M., S.C. Pratt, H.S. Seidel, and L. Kruglyak. 2006. Population genomic analysis of outcrossing and recombination in yeast. Nat. Gen. 38:1077-1081.

Sabate, J., J. Cano, A. Querol, and J.M. Guillamon. 1998. Diversity of Saccharomyces strains in wine fermentations: analysis for two consecutive years. Lett. Appl. Microbiol. 26:452-455.

Salvado, Z,, F.N. Arroyo-Lopez, J.M. Guillamon, G. Salazar, A. Querol, and E. Barrio. 2011. Temperature adaptation markedly determines evolution within the genus Saccharomyces. Appl. Environ. Microbiol. 77:2292-2302.

Schacherer, J., D.M. Ruderfer, D. Gresham, K. Dolinski, D. Botstein, and L. Kruglyak. 2007. Genome-wide analysis of nucleotide-level variation in commonly used Saccharomyces cerevisiae strains. PLoS ONE 2(3):e322.

Schacherer, J., J.A. Shapiro, D.M. Ruderfer, and L. Kruglyak. 2009. Comprehensive polymorphism survey elucidates population structure of Saccharomyces cerevisiae. Nature 458:342-345.

Schena, M., D. Shalon, R.W. Davis, and P.O. Brown. 1995. Quantitative monitoring of gene expression patterns with a complementary DNA microarray. Science 270:467-470.

Schuller, D., H. Alves, S. Dequin, and M. Casal. 2005. Ecological survey of Saccharomyces cerevisiae strains from vineyards in the Vinho Verde region of Portugal. FEMS Microbiol. Ecol. 51:167-177.

Schütz, M., and J. Gafner. 1993. Analysis of yeast diversity during spontaneous and induced alcoholic fermentations. J. Appl. Microbiol. 75:551-558

Schütz, M., and J. Gafner. 1994. Dynamics of the yeast strain population during spontaneous alcoholic fermentation determined by $\mathrm{CHEF}$ gel electrophoresis. Lett. Appl. Microbiol. 19:253-257.

Sicard, D., and J.L. Legras. 2011. Bread, beer and wine: Yeast domestication in the Saccharomyces sensu stricto complex. C.R. Biologies $334: 229-236$

Sipiczki, M. 2002. Taxonomic and physiological diversity of Saccharomyces bayanus. In Biodiversity and Biotechnology of Wine Yeasts. M. Ciani (ed.), pp. 53-69. Research Signpost, Kerala, India.

Sipiczki, M. 2008. Interspecies hybridization and recombination in Saccharomyces wine yeasts. FEMS Yeast Res. 8:996-1007.

Sipiczki, M. 2011. Diversity, variability and fast adaptive evolution of the wine yeast (Saccharomyces cerevisiae) genome-A review. Ann. Microbiol. 61:85-93.

Spor, A., T. Nidelet, J. Simon, A. Bourgais, D. de Vienne, and D. Sicard. 2009. Niche-driven evolution of metabolic and life-history strategies in natural and domesticated populations of Saccharomyces cerevisiae. BMC Evol. Biol. 9:296.

Tirosh, I., N. Sigal, and N. Barkai. 2010. Divergence of nucleosome positioning between two closely related yeast species: Genetic basis and functional consequences. Mol. Syst. Biol. 6:365.

Torija, M.J., N. Rozes, M. Poblet, J.M. Guillamon, and A. Mas. 2001. Yeast population dynamics in spontaneous fermentations: Comparison between two different wine-producing areas over a period of three years. Ant. Leeuwen. 79:345-352.

Torok, T., R.K. Mortimer, P. Romano, G. Suzzi, and M. Polsinelli. 1996. Quest for wine yeasts - An old story revisited. J. Ind. Microbiol. 17:303-313.

Townsend, J.P., D. Cavalieri, and D.L. Hartl. 2003. Population genetic variation in genome-wide gene expression. Mol. Biol. Evol. 20:955-963.

Tsai, I.J., D. Bensasson, A. Burt, and V. Koufopanou. 2008. Population genomics of the wild yeast Saccharomyces paradoxus: Quantifying the life cycle. Proc. Natl. Acad. Sci. USA 105:4957-4962.

Valero, E., B. Cambon, D. Schuller, M. Casal, and S. Dequin. 2006. Biodiversity of Saccharomyces yeast strains from grape berries of wine producing areas using starter commercial yeasts. FEMS Yeast Res. 7:317-329.

Valero, E., D. Schuller, B. Cambon, M. Casal, and S. Dequin. 2005. Dissemination and survival of commercial wine yeast in the vineyard: A large-scale, three-years study. FEMS Yeast Res. 5:959-969.

Van der Westhuizen, T.J., O.H.P. Augustyn, and I.S. Pretorius. 2000a. Geographical distribution of indigenous Saccharomyces cerevisiae strains isolated from vineyards in the coastal regions of the Western Cape in South Africa. S. Afr. J. Enol. Vitic. 21:3-9.

Van der Westhuizen, T.J., O.H.P. Augustyn, W. Kahn, and I.S. Pretorius. 2000b. Seasonal variation of indigenous Saccharomyces cerevisiae strains isolated from vineyards of the Western Cape in South Africa. S. Afr. J. Enol. Vitic. 21:10-16.

Velculescu, V.E., L. Zhang, B. Vogelstein, and K.W. Kinzler. 1995. Serial analysis of gene expression. Science 270:484-487.

Versavaud, A., P. Courcoux, C. Roulland, L. Dulau, and J.N. Hallet. 1995. Genetic diversity and geographical distribution of wild Saccharomyces cerevisiae strains from the wine-producing area of Charentes, France. Appl. Environ. Microbiol. 61:3521-3529.

Verstrepen, K.J., D. Iserentant, P. Malcorps, G. Derdelinckx, P. Van Dijck, J. Winderickx, I.S. Pretorius, J.M. Thevelein, and F.R. Delvaux. 2004. Glucose and sucrose: Hazardous fast-food for industrial yeast? Trends Biotechnol. 22:531-537.

Vezinhet, F., B. Blondin, and J.N. Hallet. 1990. Chromosomal DNA pattern and mitochondrial DNA polymorphism as toll for identification of enological strains of Saccharomyces cerevisiae. Appl. Microbiol. Biotechnol. 32:568-571.

Vezinhet, F., J.N. Hallet, M. Valade, and A. Poulard. 1992. Ecological survey of wine yeast strains by molecular methods of identification. Am. J. Enol. Vitic. 43:83-86.

Wang, S., and F. Bai. 2008. Saccharomyces arboricolus sp. Nov., a yeast species from tree bark. Int. J. Syst. Evol. Microbiol. 58:510-514.

Warringer, J., et al. 2011. Trait variation in yeast is defined by population history. PLoS Genet. 7(6):e1002111.

Wei, W., et al. 2007. Genome sequencing and comparative analysis of Saccharomyces cerevisiae strain YJM789. Proc. Natl. Acad. Sci. USA 104:12825-12830.

Winzeler, E.A., C.I. Castillo-Davis, G. Oshiro, D. Liang, D.R. Richards, Y. Zhou, and D.L. Hartl. 2003. Genetic diversity in yeast assessed with whole-genome oligonucleotide arrays. Genetics 163:79-89.

Yamamoto, N., N. Yamamoto, H. Amemiya, Y. Yokomori, K. Shimizu, and A. Totosuka. 1991. Electrophoretic karyotypes of wine yeasts. Am. J. Enol. Vitic. 42:358-363.

Yuasa, N., M. Nakagawa, M. Hahakawa, and Y. Iimura. 2004. Distribution of the sulfite resistance gene $S S U 1-R$ and the variation in its promoter region in wine yeasts. J. Biosci. Bioeng. 98:394-397. 